\title{
EFEITO DA RELAÇÃO MOLAR REDUTOR/PRECURSOR NA SÍNTESE DE NANOPARTÍCULAS DE PRATA USANDO CITRATO DE SÓDIO E DODECIL SULFATO DE SÓDIO
}

\author{
E. M. F. de $\mathrm{SÁ}^{1}$, A. BOLZAN ${ }^{1}$ e R. A. F. MACHADO ${ }^{1}$ \\ ${ }^{1}$ Universidade Federal de Santa Catarina, Departamento de Engenharia Química \\ E-mail para contato: esteladesa@gmail.com
}

\begin{abstract}
RESUMO - Neste trabalho foi realizado um estudo comparativo entre a utilização de citrato de sódio (NaCit) e dodecil sulfato de sódio (SDS) como agentes estabilizantes na síntese das nanopartículas de prata (AgNPs). As AgNPs foram obtidas através de redução química de nitrato de prata $\left(\mathrm{AgNO}_{3}\right)$ por borohidreto de sódio $\left(\mathrm{NaBH}_{4}\right)$. Tanto a relação molar entre $\mathrm{AgNO}_{3}$ e $\mathrm{NaBH}_{4}$, quanto a concentração do agente estabilizante (citrato de sódio e dodecil sulfato de sódio), foram fatores estudados empregando-se um planejamento central composto. $\mathrm{O}$ índice de polidispersidade (PDI) foi selecionado como resposta de tal planejamento, para determinação da melhor condição de síntese. A estabilidade da região de minimização da resposta (menores valores para PDI) foi avaliada, sob refrigeração, durante 45 dias. Com os dados obtidos foi possível produzir partículas com valores de PDI em torno de $20 \%$, com alta estabilidade, sem ocorrência de redução na densidade óptica, durante todo o período avaliado.
\end{abstract}

\section{INTRODUÇÃO}

A nanotecnologia é a aplicação da ciência para manipular materiais em escala atômica, a fim de criar novas estruturas de materiais, alcançando diferentes propriedades (Vaidyanathan et al., 2010). As nanopartículas de prata se destacam devido à variedade de propriedades e aplicações que possuem, tais como: condutividade elétrica, atividade biocida, efeito catalítico, etc (Keating et al., 2012). No que diz respeito à aplicação, à homogeneidade dos tamanhos das nanopartículas é um aspecto relevante e isso depende criticamente da maneira como o meio foi estabilizado e de como ocorre o crescimento das mesmas (Patakfalvi; Z. Virányi; I. Dékány, 2004). O estudo das nanopartículas leva à necessidade da compreensão das propriedades superficiais das mesmas, pois devido ao seu tamanho reduzido, as nanopartículas são instáveis por apresentarem altos valores de energia superficial. Durante a síntese as partículas tendem a se aglomerar e crescer para que, assim, ocorra diminuição na energia total do sistema (El-Shishtawy, Asiri, Al-Otaibi, 2011).

De todos os métodos bottom-up, a rota mais comum é a redução de íons prata de um sal de prata inorgânico solúvel com um agente redutor na presença de um surfactante como estabilizante (LopezMiranda, López-Valdivieso, Viramontes-Gamboa, 2012). Dodecisulfato de sódio (SDS), como um representante do grupo de surfactantes aniônicos tem sido empregados e considerados agentes estabilizantes de grande importância (Sarkar et al., 2009). Prathna, Chandrasekaran e Mukherjee (2011) estudaram a diferentes sínteses de nanopartículas coloidais nas quais usaram citrato de sódio 
como agente estabilizante, e puderam confirmar que a tendência de aglomeração para as nanopartículas recobertas por íons citrato foi diminuída. A escolha do estabilizante é crítica, pois ele determina a estabilidade, solubilidade, reatividade e, até mesmo, o tamanho e forma das nanopartículas durante a síntese (Balan et al., 2007). Nestes casos, estudar o fenômeno de superfície para nanopartículas de prata é interessante, pois é possível investigar as propriedades ópticas e àquelas dos estabilizantes adsorvidos nas nanopartículas através da absorção na região do visível devido ao plasmon de superfície (SPR - Surface Plasmon Resonance) (Kapoor, 1998).

AgNPs obtidas através da redução química do nitrato de prata por borohidreto de sódio seguem as Equações 1 e 2.

$$
\begin{aligned}
& \mathrm{AgNO}_{3}+\mathrm{NaBH}_{4} \rightarrow \mathrm{Ag}+1 / 2 \mathrm{H}_{2}+1 / 2 \mathrm{~B}_{2} \mathrm{H}_{6}+\mathrm{NaNO}_{3} \\
& \mathrm{Ag}^{+}+\mathrm{BH}_{4}^{-}+3 \mathrm{H}_{2} \mathrm{O} \rightarrow \mathrm{Ag}^{0}+\mathrm{B}(\mathrm{OH})_{3}+3,5 \mathrm{H}_{2}
\end{aligned}
$$

As partículas de prata obtidas por tal método apresentam tamanhos entre 5 a $20 \mathrm{~nm}$ de diâmetro absorção máxima, na espectroscopia UV-Vis, na faixa de 380-400 nm (Song et al., 2009), referente à banda de absorção plasmônica de superfície.

Neste trabalho foi realizado um estudo do comportamento das nanopartículas de prata, a partir da redução química em solução aquosa de nitrato de prata na presença de borohidreto de sódio, utilizando citrato de sódio ( $\mathrm{NaCit}$ ) e dodecil sulfato de sódio (SDS) como estabilizantes. As melhores condições de síntese foram determinadas empregando-se um planejamento experimental central composto, para o estudo da estabilidade sob refrigeração.

\section{PARTE EXPERIMENTAL}

\subsection{Materiais}

O nitrato de sódio, borohidreto de sódio, citrato de sódio e dodecil sulfato de sódio foram empregados em qualidade p.a. A água destilada foi usada como solvente na síntese de nanopartículas e para a preparação das soluções.

\subsection{Planejamento experimental}

Este trabalho foi realizado a partir de um planejamento experimental central composto, onde foi possível selecionar a(s) rota(s) de síntese para o estudo de estabilidade das AgNPs sob refrigeração. Os parâmetros testados foram: relação molar entre $\mathrm{NaBH}_{4}: \mathrm{AgNO}_{3}(\mathrm{mM})\left(\mathrm{x}_{1}\right)$ e concentração de citrato de sódio e dodecil sulfato de sódio $\left(\mathrm{g} . \mathrm{L}^{-1}\right)\left(\mathrm{x}_{2}\right)$. Um total de 10 experimentos foram realizados, incluindo $2^{2}=4$ pontos do cubo, 2 pontos centrais e 4 pontos axiais. Para os cálculos dos pontos axiais das variáveis $\mathrm{X}_{\mathrm{i}}$ foram codificadas como $\mathrm{x}_{\mathrm{i}}$ de acordo com a Equação 3. Os dados experimentais foram analisados usando o software Statistics (versão 7.0).

$x_{i}=\frac{X_{i}-X_{0}}{\delta X}$ 
onde $\mathrm{X}_{0}$ é o valor de $\mathrm{X}_{\mathrm{i}}$ no ponto central e $\delta \mathrm{X}$ apresenta o valor do passo (Khataee et al., 2010).

A análise estatística dos valores médios obtidos nas caracterizações das rotas de sínteses na região de mínimo foi realizada por Análise de Variância (ANOVA). O teste de Tukey foi aplicado quando verificada diferença significativa de $5 \%$ entre os valores, utilizando o software OriginLab ${ }^{\circledR}$ (versão 7.5).

\subsection{Síntese e caracterização das nanopartículas de prata}

As nanopartículas de prata foram sintetizadas a partir de redução química de soluções aquosas nitrato de prata $(1 \mathrm{mM})$ com borohidreto de sódio, de acordo com as variáveis selecionadas no planejamento anterior.

A caracterização dos parâmetros de ressonância plasmônica de superfície, que correspondem ao comprimento de onda no máximo de absorção $\left(\lambda_{\text {máx }}\right)$, valor máximo de absorção (SPR) e largura à meia altura (FWHM) foram monitoradas por espectrofotômetro UV-Vis (Hitach U-1900), utilizando modo de varredura em comprimentos de onda de 300 a $600 \mathrm{~nm}$. A caracterização, em termos de Zmédio, índice de polidispersidade (PDI) $(\%)$ e potencial zeta $(\mathrm{mV})$ das AgNPs, foi realizada através de análise de espalhamento de luz (DLS), utilizando NanoSizer (Malvern Instruments Nano-S-ZEN 1600). As AgNPs com e sem estabilizantes foram armazenadas sob refrigeração em frasco âmbar durante 45 dias para a avaliação da estabilidade, medida realizada em diferentes períodos $(0,7,15$ e 45 dias) por espectroscopia UV-Vis (Hitachi U-1900).

\section{RESULTADOS E DISCUSSÃO}

O parâmetro de polidispersidade (PDI) das AgNPs foi selecionado como resposta (Y) para obter-se a melhor rota de síntese, utilizando tanto o NaCit quanto o SDS, para o estudo da estabilidade sob refrigeração. Portanto, para estimar a resposta, um modelo empírico polinomial de segunda ordem foi obtido para cada estabilizante utilizado. O modelo para a utilização do citrato de sódio é apresentado na Equação 3, e, para a utilização do dodecil sulfato de sódio é apresentado na Equação 4. Os fatores e os níveis dos mesmos são apresentados na Tabela 1.

$$
\begin{aligned}
& P D I=0,27-0,09 M+0,09 E-0,13 M E+0,07 M^{2}-0,03 E^{2} \\
& P D I=0,22-0,05 M-0,12 E-0,05 M E+0,09 M^{2}+0,06 E^{2}
\end{aligned}
$$

onde PDI é o índice de polidispersidade, $M$ é a relação molar entre $\mathrm{NaBH}_{4}: \mathrm{AgNO}_{3}$ e $E$ é a concentração do estabilizante $\left(\mathrm{g} . \mathrm{L}^{-1}\right)$.

No planejamento experimental fatorial foram investigados os efeitos principais de todos os fatores experimentais de interesse e os efeitos de interação destes fatores na resposta desejada. Através da análise de regressão e da análise de variância (ANOVA), foi possível observar a significância estatística tanto para o modelo quando aplicou-se o NaCit $\left(\mathrm{R}^{2}=0,9375\right)$ quanto para a aplicação do $\operatorname{SDS}\left(\mathrm{R}^{2}=0,9548\right)(\mathrm{p}<0,05)$, através do cálculo de coeficiente de variação $(\mathrm{CV} \%)$ e erro 
percentual (Erro \%), pelo fato de que os valores preditos se aproximaram dos valores experimentais demonstrando que o modelo foi aplicável (Tabela 2).

Tabela 1 - Condições estabelecidas pelo planejamento experimental

\begin{tabular}{|c|c|c|c|c|c|}
\hline Fatores & $\begin{array}{c}-\alpha \\
(-1,41)\end{array}$ & -1 & 0 & +1 & $\begin{array}{c}+\alpha \\
(+1,41) \\
\end{array}$ \\
\hline Relação molar $\mathrm{NaBH}_{4}: \mathrm{AgNO}_{3}$ & 0,2 & 1 & 3 & 5 & 6 \\
\hline Concentração do estabilizante (g.L $\left.\mathrm{L}^{-1}\right)$ & 0,12 & 0,25 & 1,50 & 2,20 & 2,88 \\
\hline
\end{tabular}

Tabela 2 - Condições de síntese das AgNPs utilizando NaCit e SDS como estabilizantes ${ }^{1}$

\begin{tabular}{|c|c|c|c|c|c|c|c|}
\hline \multirow[b]{2}{*}{ Experimentos } & \multicolumn{2}{|c|}{ Fatores } & \multicolumn{5}{|c|}{ Valores Respostas para NaCit } \\
\hline & $\begin{array}{c}\mathrm{NaBH}_{4}: \mathrm{AgNO}_{3} \\
(\mathrm{mM})\end{array}$ & $\begin{array}{l}\text { NaCit } \\
\left(\mathrm{g} \cdot \mathrm{L}^{-1}\right)\end{array}$ & $\begin{array}{c}\text { PDI } \\
\text { Experimental }\end{array}$ & $\begin{array}{l}\mathrm{CV} \\
(\%)\end{array}$ & $\begin{array}{c}\text { PDI } \\
\text { Predito }\end{array}$ & $\begin{array}{l}\mathrm{CV} \\
(\%)\end{array}$ & $\begin{array}{l}\text { Erro } \\
(\%)\end{array}$ \\
\hline 1 & 1 & 0,25 & $0,12 \pm 0,01$ & 4,0 & $0,12 \pm 0,01$ & 4,9 & 3,2 \\
\hline 2 & 1 & 2,20 & $0,57 \pm 0,32$ & 56,8 & $0,62 \pm 0,33$ & 52,9 & 9,7 \\
\hline 3 & 5 & 0,25 & $0,26 \pm 0,11$ & 41,6 & $0,24 \pm 0,10$ & 42,5 & 7,8 \\
\hline 4 & 5 & 2,20 & $0,22 \pm 0,09$ & 40,8 & $0,18 \pm 0,09$ & 47,5 & 16,5 \\
\hline 5 & 0,2 & 1,50 & $0,69 \pm 0,37$ & 52,8 & $0,67 \pm 0,10$ & 15,4 & 3,5 \\
\hline 6 & 6 & 1,50 & $0,23 \pm 0,17$ & 73,0 & $0,27 \pm 0,07$ & 27,6 & 15,0 \\
\hline 7 & 3 & 0,12 & $0,17 \pm 0,03$ & 17,9 & $0,17 \pm 0,01$ & 54,9 & 1,2 \\
\hline 8 & 3 & 2,88 & $0,33 \pm 0,25$ & 76,4 & $0,32 \pm 0,05$ & 16,3 & 3,6 \\
\hline 9 & 3 & 1,50 & $0,28 \pm 0,07$ & 24,4 & $0,29 \pm 0,11$ & 35,6 & 5,3 \\
\hline \multirow[t]{2}{*}{10} & 3 & 1,50 & $0,29 \pm 0,10$ & 34,7 & $0,29 \pm 0,03$ & 8,4 & 2,4 \\
\hline & \multicolumn{2}{|c|}{ Fatores } & \multicolumn{5}{|c|}{ Valores Respostas para SDS } \\
\hline Experimentos & $\begin{array}{c}\mathrm{NaBH}_{4}: \mathrm{AgNO}_{3} \\
(\mathrm{mM})\end{array}$ & $\begin{array}{c}\text { SDS } \\
\left(\mathrm{g} \cdot \mathrm{L}^{-1}\right)\end{array}$ & $\begin{array}{c}\text { PDI } \\
\text { Experimental }\end{array}$ & $\begin{array}{l}\mathrm{CV} \\
(\%)\end{array}$ & $\begin{array}{c}\text { PDI } \\
\text { Predito }\end{array}$ & $\begin{array}{l}\mathrm{CV} \\
(\%)\end{array}$ & $\begin{array}{l}\text { Erro } \\
(\%) \\
\end{array}$ \\
\hline 1 & 1 & 0,25 & $0,41 \pm 0,19$ & 46,2 & $0,48 \pm 0,01$ & 20,6 & 17,4 \\
\hline 2 & 1 & 2,20 & $0,35 \pm 0,09$ & 25,3 & $0,35 \pm 0,11$ & 31,9 & 1,1 \\
\hline 3 & 5 & 0,25 & $0,49 \pm 0,04$ & 7,3 & $0,51 \pm 0,16$ & 31,6 & 3,3 \\
\hline 4 & 5 & 2,20 & $0,29 \pm 0,01$ & 4,1 & $0,28 \pm 0,04$ & 15,6 & 3,1 \\
\hline 5 & 0,2 & 1,50 & $0,50 \pm 0,10$ & 20,3 & $0,54 \pm 0,11$ & 20,8 & 7,9 \\
\hline 6 & 6 & 1,50 & $0,21 \pm 0,09$ & 43,7 & $0,22 \pm 0,08$ & 34,2 & 6,3 \\
\hline 7 & 3 & 0,12 & $0,51 \pm 0,20$ & 38,9 & $0,43 \pm 0,10$ & 23,6 & 15,1 \\
\hline 8 & 3 & 2,88 & $0,15 \pm 0,02$ & 10,3 & $0,20 \pm 0,09$ & 43,6 & 38,3 \\
\hline 9 & 3 & 1,50 & $0,15 \pm 0,07$ & 48,7 & $0,19 \pm 0,02$ & 10,0 & 22,7 \\
\hline 10 & 3 & 1,50 & $0,16 \pm 0,07$ & 46,2 & $0,19 \pm 0,05$ & 28,6 & 19,6 \\
\hline
\end{tabular}

${ }^{5}$ Os valores obtidos para o Z-médio refere-se ao valor médio obtido das medidas em triplicata.

As rotas de síntese que apresentaram menores valores de índice de polidispersidade, para o planejamento experimental desenvolvido no estudo utilizando citrato de sódio como estabilizante, foram obtidas em três condições como destacado na Figura 1, através dos gráficos de superfície de resposta (A) e os contornos, para o modelo de regressão de dados nas diferentes condições do 
planejamento experimental. Tais regiões que são correspondentes aos experimentos 1, 4 e 7 (Tabela 2), nas quais a relação entre $\mathrm{NaBH}_{4}: \mathrm{AgNO}_{3}$ variaram de 1,5 e $3 \mathrm{mM}$ e a concentração de $\mathrm{NaCit}$ variou de $0,25,2,20$ e 0,12 g. $\mathrm{L}^{-1}$, respectivamente. Em relação à utilização do SDS como estabilizante, a resposta mínima de índice de polidispersidade das AgNPs foi obtida nas condições correspondentes ao experimento 4 (Tabela 2), tal região é destacada na Figura 2 e identificam a variação da relação entre $\mathrm{NaBH}_{4}: \mathrm{AgNO}_{3}$ sendo $5 \mathrm{mM}$, e a concentração de 2,20 g.L ${ }^{-1}$ de $\mathrm{SDS}$.
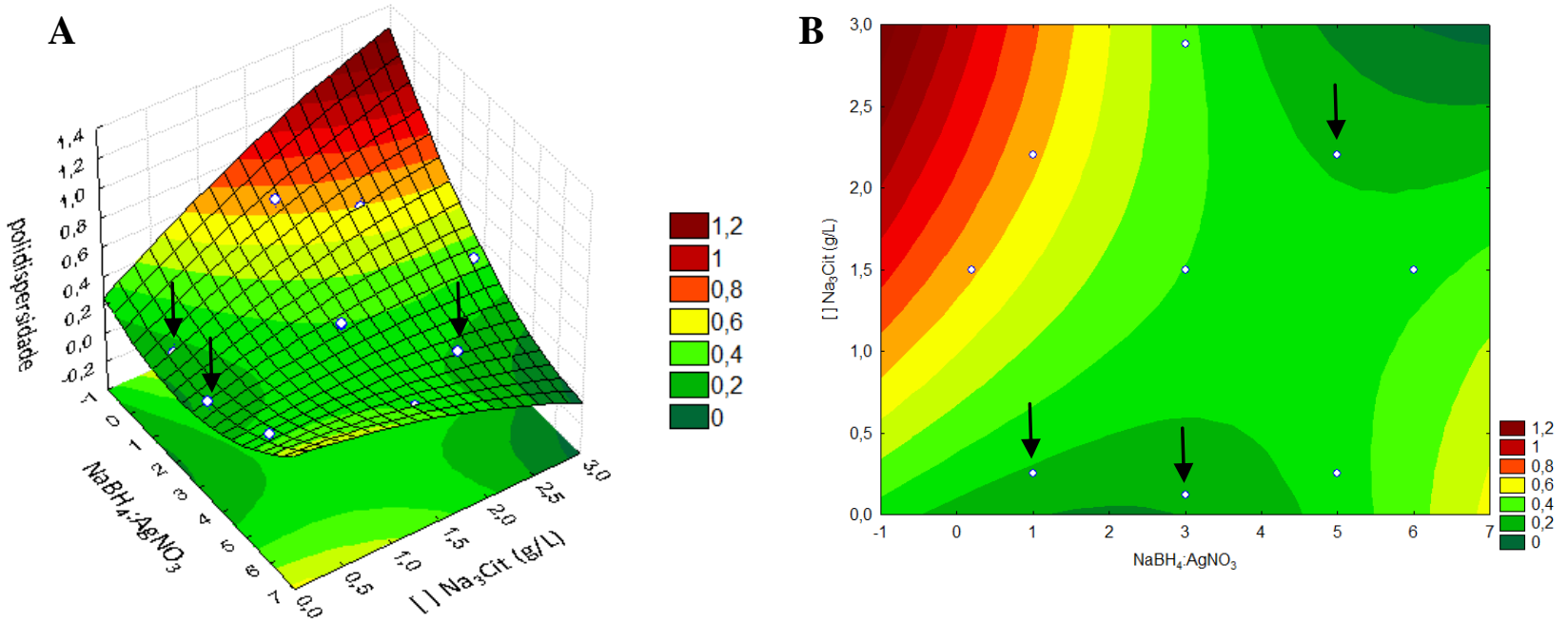

Figura 1 - Relação entre concentração de $\mathrm{NaCit}$ e relação molar $\mathrm{NaBH}_{4}: \mathrm{AgNO}_{3}$ em função da polidispersidade. Gráfico da superfície de resposta (A) e de contorno (B).
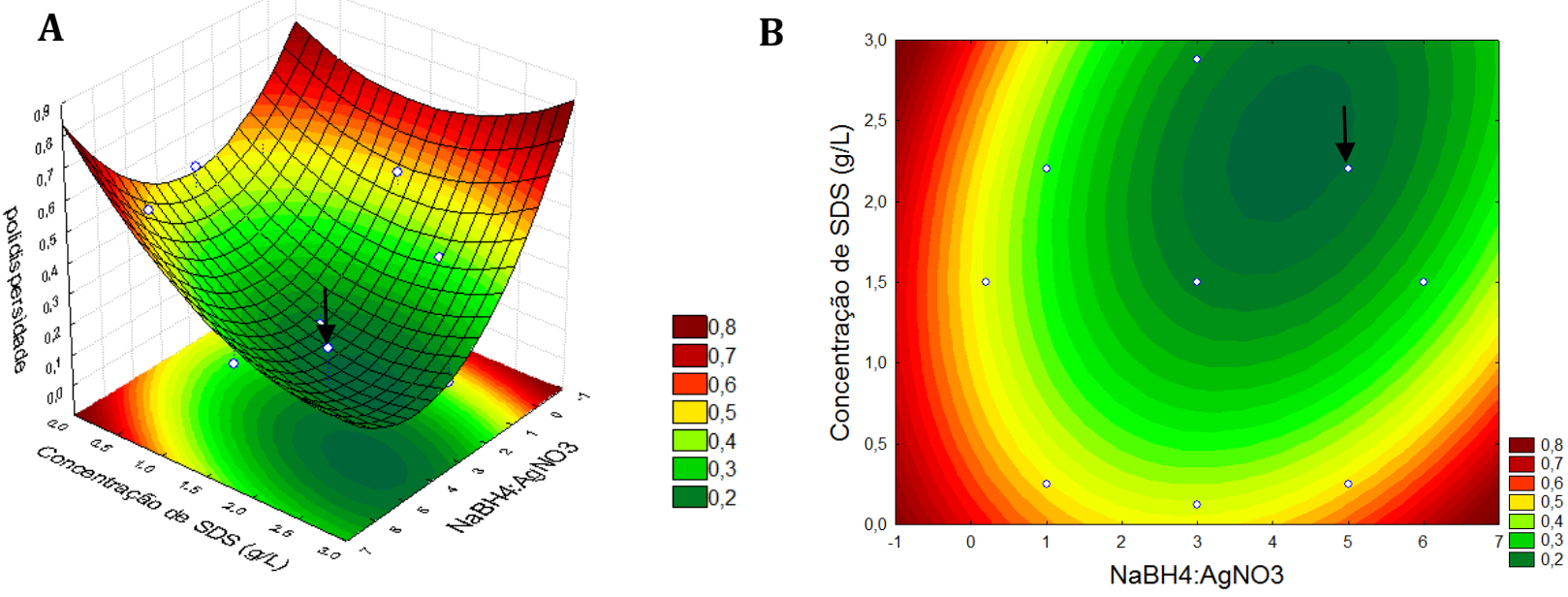

Figura 2 - Relação entre concentração de SDS e relação molar $\mathrm{NaBH}_{4}: \mathrm{AgNO}_{3}$ em função da polidispersidade. Gráfico da superfície de resposta (A) e de contorno (B).

Considerando a região de mínimo obtidas para a utilização tanto do NaCit quanto do SDS como estabilizantes, pode-se selecionar tais condições experimentais, as quais foram caracterizadas em 


\section{9 a 22 de outubro de 2014 \\ Florianópolis/SC}

termos de Z-médio $(\mathrm{nm})$, índice de polidispersidade $(\%)$, potencial zeta $(\mathrm{mV})$ e parâmetros de ressonância plasmônica de superfície, como apresentado na Tabela 3.

Tabela 3 - Caracterizações das rotas de síntese de AgNPs selecionadas nos planejamento experimentais correspondente ao menor índice de polidispersidade ${ }^{1}$

\begin{tabular}{|c|c|c|c|c|c|c|}
\hline $\begin{array}{l}\text { Experimento/ } \\
\text { Estabilizante }\end{array}$ & $\begin{array}{l}\text { Z-Médio } \\
\text { (nm) }\end{array}$ & $\begin{array}{c}\mathrm{PDI}^{3} \\
(\%)\end{array}$ & $\begin{array}{l}\text { Caract } \\
\text { Ressonância } \\
\mathrm{A}_{\text {máx }}{ }^{4}\end{array}$ & $\begin{array}{l}\text { zações } \\
\text { lasmônica } \\
\text { SPR }^{5} \\
(\mathrm{~nm})\end{array}$ & $\begin{array}{l}\text { Superfície } \\
\text { FWHM }^{6} \\
(\mathrm{~nm})\end{array}$ & $\begin{array}{c}\text { Potencial } \\
\text { Zeta } \\
(\mathrm{mV})\end{array}$ \\
\hline Controle $^{2}$ & $112,0 \pm 1,0^{\mathrm{a}}$ & $25,2 \pm 0,9^{\mathrm{a}}$ & $1,24 \pm 0,52^{\mathrm{a}}$ & $386 \pm 1,0^{\mathrm{a}}$ & $88,9 \pm 0,9^{\mathrm{a}}$ & $-25,9 \pm 0,5^{\mathrm{ac}}$ \\
\hline 1/NaCit & $58,5 \pm 0,8^{\mathrm{b}}$ & $12,6 \pm 0,3^{\mathrm{b}}$ & $1,49 \pm 0,10^{\mathrm{ab}}$ & $393 \pm 1,0^{b}$ & $70,3 \pm 0,5^{\mathrm{b}}$ & $-35,8 \pm 1,6^{b}$ \\
\hline 4/NaCit & $74,1 \pm 1,2^{\mathrm{c}}$ & $22,8 \pm 0,6^{\mathrm{c}}$ & $1,45 \pm 0,65^{\mathrm{ab}}$ & $393 \pm 1,0^{b}$ & $74,0 \pm 0,6^{\mathrm{c}}$ & $-23,0 \pm 0,5^{\mathrm{a}}$ \\
\hline 7/NaCit & $68,8 \pm 0,7^{\mathrm{d}}$ & $17,3 \pm 0,4^{\mathrm{d}}$ & $1,55 \pm 0,51^{\mathrm{ab}}$ & $391 \pm 0,6^{\mathrm{b}}$ & $74,9 \pm 0,8^{\mathrm{c}}$ & $-28,6 \pm 0,4^{\mathrm{cd}}$ \\
\hline 4/SDS & $74,0 \pm 0,1^{\mathrm{c}}$ & $20,0 \pm 0,4^{\mathrm{e}}$ & $2,63 \pm 0,18^{\mathrm{b}}$ & $393 \pm 0,6^{\mathrm{b}}$ & $81,4 \pm 0,4^{\mathrm{d}}$ & $-30,5 \pm 1,8^{\mathrm{d}}$ \\
\hline
\end{tabular}

${ }^{\mathrm{I}}$ Valores dentro da coluna não distribuídos com mesma letra são significativamente diferentes $(\mathrm{p}<0,05)$ por teste de separação de médias de Tukey, sendo a relação entre os experimentos. ${ }^{2}$ Amostra sem estabilizante; ${ }^{3}$ índice de polidispersidade; ${ }^{4}$ valor máximo de absorbância; ${ }^{5}$ pico de ressonância plasmônica de superfície; ${ }^{6}$ largura à meia-altura referente ao valor máximo de absorção óptica.

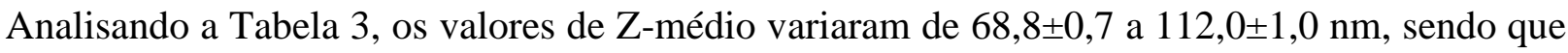
diferença significativa $(\mathrm{p}<0,05)$ foi observada entre os experimentos em relação à utilização do estabilizante. Ou seja, a adição de estabilizante, possivelmente, interferiu na redução do tamanho médio das AgNPs, e a utilização de citrato de sódio na concentração de $0,25 \mathrm{~g} . \mathrm{L}^{-1}$, com a relação $\mathrm{NaBH}_{4}: \mathrm{AgNO}_{3}$ de $1 \mathrm{mM}$ permitiu a obtenção de $\operatorname{AgNPs}$ menores $(58,5 \pm 0,8)$ e menos polidispersas $(12,6 \pm 0,3 \%)$. Dentre os parâmetros da região de Ressonância Plasmônica de Superfície, foi observada diferença significativa no parâmetro FWHM, que, portanto, confirma o menor grau de dispersão do tamanho de nanopartículas $(70,3 \pm 0,5)$ para o experimento $1 / \mathrm{NaCit}$. Observou-se que a adição dos estabilizantes deslocou o pico SPR para comprimentos de onda maiores, o que, segundo Pal, Sau e Jana (1997), representa a ocorrência da remoção de densidade de elétrons da superfície das nanopartículas, associada com a adição das moléculas dos estabilizantes.

Agentes fortemente redutores como o borohidreto de sódio, induzem a formação de centros de nucleação que crescem formando pequenos clusters, cujo tamanho final, depende dos fatores reacionais (Solomon et al., 2007). Observou-se que o valor máximo de absorbância variou significativamente para as AgNPs que foram estabilizadas por SDS. Segundo Gorup (2010), o valor da intensidade de máxima absorção varia linearmente com a densidade dos clusters produzidos durante a síntese das nanopartículas ou do próprio número de partículas/volume. No caso da adição de SDS, o aumento do valor máximo de absorbância, pode estar relacionado às características de tamanho e dispersão das nanopartículas, que se apresentaram melhores em relação ao experimento controle (sem estabilizante). Mas, o aumento significativo do parâmetro $\mathrm{A}_{\text {máx, pode estar também }}$ relacionado à uma saturação na interação da nanopartícula e do SDS, quando comparado com o

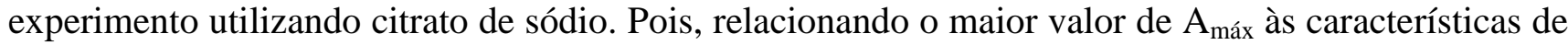
dispersão e tamanho das partículas, a partir do os valores de Z-médio, PDI e FWHM para a utilização do SDS, foram significativamente maiores ao comparar-se à utilização do NaCit. 
Foram observadas diferenças significativas $(\mathrm{p}<0,05)$ para os valores de potencial zeta, e como resultado da adsorção tanto do NaCit quanto do SDS na superfície das AgNPs, os valores para este parâmetro aumentaram negativamente, como tem sido demonstrado por Hedberg et al. (2012), que contribuiu para a estabilização das nanopartículas quando comparadas com o experimento controle.

A estabilidade das soluções de AgNPs foi avaliada durante 45 dias sob refrigeração, e os perfis foram traçados em diferentes períodos por espectroscopia de UV-Vis para os estabilizantes utilizados neste trabalho. Na Figura 3, são apresentados os espectros que caracterizam as AgNPs durante o período do estudo da estabilidade sob refrigeração. Inicialmente, é possível observar, que o experimento controle é extremamente instável, pois o pico de absorção plasmônica reduziu gradativamente ao longo do período de armazenamento. O comprimento de onda variou de 386 $\mathrm{nm}$ a $395 \mathrm{~nm}$. A redução total na densidade óptica aos 45 dias de armazenamento foi de aproximadamente $62 \%$ e a largura à meia altura aumentou aproximadamente $3 \%$. Comparando o controle com a utilização do citrato de sódio (Figura 3A), é possível observar que, o valor de densidade óptica não sofreu redução após 45 dias. Portanto, este comportamento demonstra a eficiência do citrato de sódio em estabilizar as AgNPs por este período de tempo. Referente às condições utilizando SDS como estabilizante (Figura 3 B), foi possível observar que ao longo do período de armazenamento das AgNPs, a densidade óptica reduziu 2,5\% e a largura à meia altura aumentou em torno de 5\%. Os valores de comprimento de onda variaram de 393 a $400 \mathrm{~nm}$.
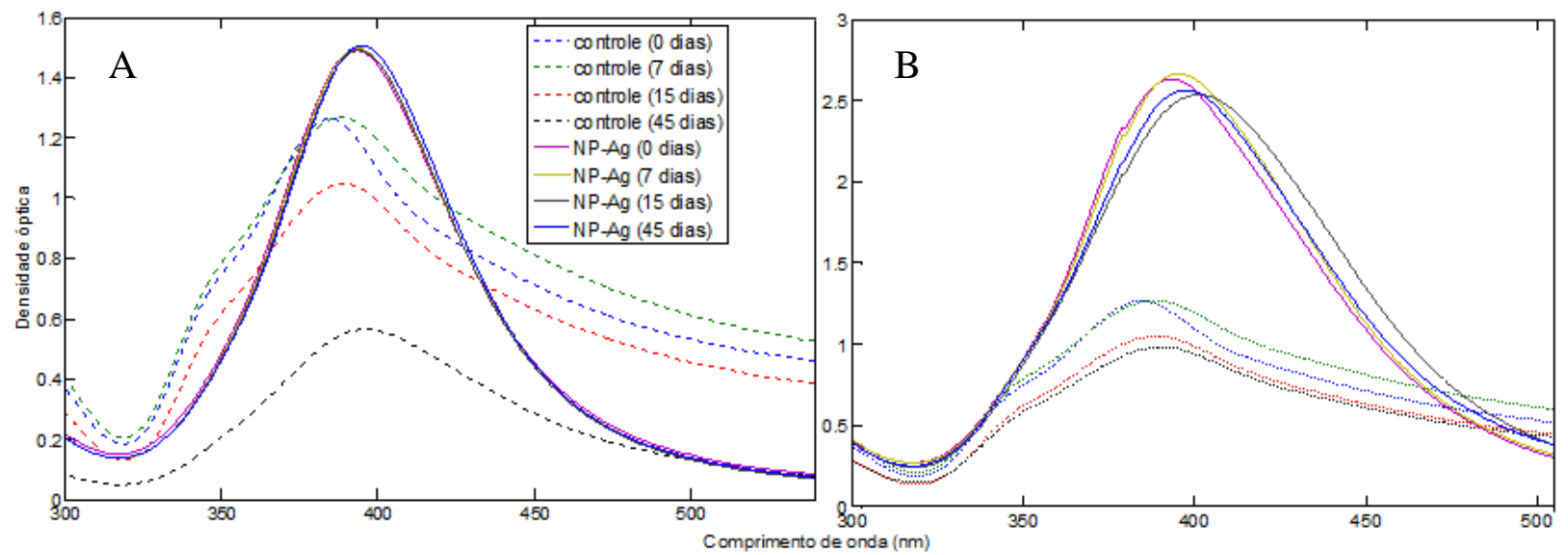

Figura 3 - Espectros UV-Vis das AgNPs durante o teste de estabilidade. AgNP estabilizadas com NaCit (A) e com SDS (B).

\section{CONCLUSÃO}

Diante destes espectros de UV-Visível ao longo de 45 dias e das demais caracterizações (Tabela 3) é possível afirmar que, a utilização do citrato de sódio, com as condições estabelecidas no experimento 1 do respectivo planejamento experimental, parecem ser as condições mais viáveis em termos de estabilidade das AgNPs.

\section{REFERÊNCIAS}




\section{9 a 22 de outubro de 2014 \\ Florianópolis/SC}

BALAN, L.; MALVAL, J-P; SCHNEIDER, R.; BURGET, D. Silver nanoparticles: new synthesis, characterization and photophysical properties. Materials Chemistry and Physics, v.104, p.417-421, 2007.

EL-SHISHTAWY, R.; ASIRI, A.M.; AL-OTAIBI, M.M. Synthesis and spectroscopic studies of stable aqueous dispersion of silver nanoparticles. Spectrochimia Acta Part A, v. 79, p. 15051510, 2011.

GORUP, L.F. Nanopartículas coloidais de ouro e prata e sua funcionalização com dibutildicalcogenetos. São Carlos: UFSCar, 2010.

HEDBERG, J. et al. Interactions between surfactants and silver nanoparticles of variyng charge. J. Colloid Interface Sci. v. 369, p. 193-201, 2012.

KAPOOR, S. Preparation, characterization, and surface modification of silver particles. Langmuir, v. 14, p. 1021-1025, 1998.

KEATING, M.; CHEN, Y.; LARMOUR, I.A.; FAULDS, K.; GRAHAM, D. Growth and surfaceenhanced Raman scattering of Ag nanoparticle assembly in agarose gel.Measurement Science and Technology, 23, p.1-9, 2012.

LÓPEZ-MIRANDA, A., LÓPEZ-VALDIVIESO, A., VIRAMONTES-GAMBOA, G. Silver nanoparticles synthesis in aqueous solutions using sulfite as reducing agent and sodium dodecyl sulfate as stabilizer. J. Nanopart. Res., v.14, p. 1-11, 2012.

PAL, T., SAU, T.K., JANA, N.R. Reversible Formation and Dissolution of Silver Nanoparticles in Aqueous Surfactant Media. v. 13, p 1481-1485, 1997.

PATAKFALVI, R.; VIRÁNYI, Z.; DÉKÁNY, I. Kinetics of silver nanoparticles growth in aqueous polymer solutions.Colloid Polymers Science, v. 283, p. 299-305, 2004.

PRATHNA, T.C.; CHANDRASEKARAN, N.; MUKHERJEE, A. Studies on aggregation behavior of silver nanoparticles in aqueous matrices: Effect of surface functionalization and matrix composition. Colloids and Surfaces A: Physicochemical Engineering Aspects, v.390, p. 216-224, 2011.

SARKAR, A.; CHADHA, R.; BISWAS, N.; MUKHERJEE, T.; KAPOOR, S. Phase-transfer and film formation of silver nanoparticles.Journal of Colloid and Interface Science, v.332, p. 224230, 2009.

SOLOMON, S. D.; BAHADORY, M.; JEYARAJASINGAM, A.V.; RUTKOWSKY, S.A.; BORITZ, C. Synthesis and Study of Silver Nanoparticles.Journal of Chemical Education, v. 84, n. 2, p. 322-325, 2007.

SONG, K.C.; LEE, S.M.; PARK, T.S.; LEE, B.S. Preparation of colloidal silver nanoparticles by chemical reduction method. Korean J. Chem. Eng., v.26(1), p.153-155, 2009.

VAIDYANATHAN, R., GOPALRAM, S., KALISHWARALAL, K., DEEPAK, V., PANDIAN, S. R. K., e GURUNATHAN, S. Enhanced silver nanoparticle synthesis by optimization of nitrate reductase activity. Colloids and Surfaces B: Biointerfaces, 75(1), p. 335-341, 2010. 